\title{
Comparing the American and European diagnostic guidelines for cystic fibrosis: same disease, different language?
}

\author{
Chee Y Ooi, ${ }^{1,2,3}$ Annie Dupuis, ${ }^{4,5}$ Lynda Ellis, ${ }^{3}$ Keith Jarvi, ${ }^{6}$ Sheelagh Martin, ${ }^{3}$ \\ Tanja Gonska, ${ }^{2,3}$ Ruslan Dorfman, ${ }^{7}$ Paul Kortan, ${ }^{8}$ Melinda Solomon, ${ }^{2,9}$ \\ Elizabeth Tullis, ${ }^{8,10}$ Peter R Durie ${ }^{2,3}$
}

- Additional tables are published online only. To view these files please visit the journal online (http://thorax.bmj. com/content/67/7.toc).

'School of Women's and Children's Health, Faculty of Medicine, University of New South Wales and Sydney Children's Hospital, Randwick, Sydney, Australia ${ }^{2}$ Physiology and Experimental Medicine, Research Institute, Hospital for Sick Children, Toronto, Ontario, Canada ${ }^{3}$ Division of Gastroenterology, Hepatology and Nutrition, Department of Paediatrics, Hospital for Sick Children, Toronto, Ontario, Canada ${ }^{4}$ Child Health Evaluative Sciences, Hospital for Sick Children, Toronto, Ontario, Canada

${ }^{5}$ Dalla Lana School of Public Health, University of Toronto, Toronto, Ontario, Canada ${ }^{6}$ Division of Urology, Mount Sinai Hospital, Toronto, Ontario, Canada

${ }^{7}$ Genetics and Genome Biology, Research Institute, Hospital for Sick Children, Toronto, Ontario, Canada

${ }^{8}$ Department of Medicine, University of Toronto, Toronto, Ontario, Canada

${ }^{9}$ Division of Respirology, Department of Paediatrics, Hospital for Sick Children, Toronto, Ontario, Canada ${ }^{10}$ Division of Respirology, Keenan Research Centre of Li Ka Shing Knowledge Institute, St Michael's Hospital, Toronto Ontario, Canada

\section{Correspondence to}

Dr (Keith) Chee Y Ooi, Sydney Children's Hospital Randwick,

School of Women's and Children's Health, High Street, Randwick, NSW 2031, Australia;

keith.ooi@unsw.edu.au

Received 1 December 2011 Accepted 22 March 2012 Published Online First

15 April 2012

\section{ABSTRACT}

Background The American and European cystic fibrosis (CF) guidelines recommend different diagnostic criteria. This study assessed diagnostic concordance between these recommendations.

Methods Subjects with single organ manifestations suggestive of CF (chronic sinopulmonary disease (RESP), chronic/recurrent pancreatitis (PANC) or obstructive azoospermia (AZOOSP)) were prospectively evaluated by sweat test, nasal potential difference and genotyping. Concordance in diagnostic outcomes between the two algorithms was measured using observed agreement and $\mathrm{K}$ statistics.

Results A total of 208 subjects were evaluated. Observed agreement was $84.8 \%$ and level of agreement was excellent ( $\kappa=0.87$ ) between the American and European recommendations. The RESP phenotype was associated with the highest degree of concordance (observed agreement $\geq 90 \%, \kappa=0.92$ ) compared with the PANC (observed agreement $86 \%, \kappa=0.65$ ) and AZOOSP (observed agreement $80 \%, \kappa=0.87$ ) phenotypes. Incorporation of nasal potential difference into the American algorithm failed to improve the overall degree of concordance (good agreement level; $\kappa=0.75$ ); the level of agreement was unchanged in RESP and PANC subjects, but reduced in AZOOSP subjects (from excellent to good). Extensive genotyping had limited clinical utility in the diagnosis of CF in both algorithms. Conclusions Despite inconsistencies between the American and European diagnostic recommendations, concordance in diagnostic outcomes among subjects presenting with single organ manifestations of CF was good to excellent. These diagnostic guidelines provide guidance and promote rigorous evaluation for the diagnosis of CF but neither guideline should be regarded as dogma.

\section{INTRODUCTION}

Cystic fibrosis (CF) was previously thought to be a multisystem disease that manifests either at birth (with intestinal obstruction) or in infancy/early childhood (with growth failure and recurrent sinopulmonary symptoms). It is now recognised that a broad spectrum of conditions are associated with mutations in the CF transmembrane conductance regulator (CFTR) gene. This includes older children and adults presenting with manifestations in one organ, including sino-pulmonary diseases, pancrea-

\section{Key messages}

What is the key question?

- Do individuals with single organ manifestations of cystic fibrosis (CF) have different diagnostic outcomes when assessed by American or European diagnostic guidelines for CF?

What is the bottom line?

- Overall, there was 'good to excellent' concordance between the American and European diagnostic algorithms for CF.

\section{Why read on?}

- Approximately $15 \%$ of patients had discordant diagnostic outcomes, a third of which were related to different cutoffs for borderline sweat chloride concentration ( 30 vs $40 \mathrm{mmo} / /$ litre). Concordance was not improved when adjunctive nasal potential difference testing was included in the American algorithm.

titis or obstructive azoospermia. ${ }^{1-6}$ In many of these individuals, a diagnosis of CF is difficult to establish or exclude.

The US CF Foundation and European CF Society each convened expert panels to establish consensus on the diagnostic criteria for $\mathrm{CF}^{7} 8$ Different terminologies and the lack of objective evidence to support or counter expert opinions resulted in differences in the application and interpretation of diagnostic tests. The American report recommended that diagnostic terminology be limited to 'CF', 'CFTR-related disorder' or 'unlikely CF'. The European guidelines label individuals as having 'classic CF', 'CFTR dysfunction' (within which 'non-classic/atypical CF' or an item from the WHO diagnostic list can be ascribed), 'inconclusive' or 'unlikely CF'.

For initial testing, the American guidelines recommended sweat chloride with CFTR genotyping, whereas the European guidelines begin with sweat chloride or CFTR genotyping. The European guideline recommends sweat chloride of $30 \mathrm{mmol} /$ litre as the lower cutoff for the intermediate range at all ages. However, the American guideline uses $30 \mathrm{mmol} /$ litre as the cutoff up to $6 \mathrm{months}$ of age; thereafter the cutoff is raised to $40 \mathrm{mmol} /$ litre. $^{.} 8$ 
Both reports recommended initial genotyping using a limited CFTR mutation panel. The American report limited the list to 23 mutations that were established by the American College of Medical Genetics (ACMG). ${ }^{9}$ In contrast, the European algorithm recommended mutations that reflect the distribution and frequency of the local population and classified patients according to the number of CFTR mutations identified (0, 1 or 2) without providing guidance concerning their consequences (CF-causing vs CFTR-related disorder vs no consequence). Both guidelines reserve extensive genotyping for cases with diagnostic uncertainty.

The two reports offer differing recommendations concerning the role of ancillary tests, such as the nasal potential difference (NPD) test. The American report recommended that NPD be used to provide supportive evidence of CF when a diagnosis remains uncertain. The European report incorporated NPD into its diagnostic algorithm, thereby advocating it as the definitive test in cases of diagnostic uncertainty. ${ }^{10}$ Neither report offered recommendations concerning standard operating procedures and diagnostic reference values.

We tested concordance between the European and American recommendations by evaluating the diagnostic outcomes of prospectively ascertained, undiagnosed individuals, referred to the Toronto CF clinics with single organ manifestations of CF.

\section{METHODS \\ Study population}

This study was approved by the research ethics boards of all participating institutions (\#0020020091 Hospital for Sick Children, \#02-156 St Michael's Hospital and \#03-0084-E Mt Sinai Hospital). Written consent was obtained from all subjects.

Undiagnosed individuals with single organ manifestations of CF were prospectively and consecutively enrolled (1994-2008) into a study cohort designed to re-evaluate the diagnostic parameters of CF disease. This included subjects with idiopathic chronic sinopulmonary disease (RESP), idiopathic recurrent, acute or chronic pancreatitis (PANC) or men with infertility due to obstructive azoospermia (AZOOSP). Idiopathic sinopulmonary disease was defined as recurrent or chronic sinusitis (including sinusoidal pain, nasal discharge, postnasal drip), nasal polyps, recurrent or chronic bronchitis, recurrent pneumonia and/or bronchiectasis for at least 6 months. All enrolled subjects with sinopulmonary disease had three or more of these symptoms. If not done prior to referral, RESP subjects were tested for immunodeficiency, $\alpha$-1-antitrypsin deficiency, allergic bronchopulmonary aspergillosis, non-tuberculous mycobacteria, and primary ciliary dyskinesia. Patients were also screened for conditions known to be associated with bronchiectasis (eg, rheumatoid arthritis, other collagen vascular diseases and inflammatory bowel disease). Patients diagnosed as having any of these disorders were excluded from the study. A diagnosis of idiopathic recurrent acute pancreatitis was accepted following at least two episodes of abdominal pain associated with raised serum amylase and/or lipase (more than two times the upper limit of the reference range), and/or imaging evidence of acute pancreatitis such as pancreatic oedema, haemorrhage or necrosis. Patients with chronic pancreatitis had chronic pain in association with pancreatic calcifications and/or characteristic ductal changes. A diagnosis of obstructive azoospermia (congenital unilateral or bilateral absence of vas deferens) was confirmed by physical examination, transrectal ultrasound and evidence of azoospermia on two separate occasions. No patients were excluded on the basis of sex or race (defined by patient self- report). Exocrine pancreatic function was performed using one or more tests, including $72 \mathrm{~h}$ faecal fat, faecal elastase-1, and/or serum cationic trypsinogen. Seventeen PANC, 60 AZOOSP and 72 RESP subjects have been reported elsewhere in a different context. 3611

\section{Ion channel measurements (sweat test and NPD)}

Sweat testing (Gibson and Cooke ${ }^{12}$ (before 2005) or Macroduct $^{13}$ methods) and NPD were performed on the same day. NPD was performed according to Knowles and colleagues by a single operator masked to other test results. ${ }^{14}$ The change in CFTR-mediated chloride diffusion following perfusion with a chloride-free solution and isoproterenol ( $\Delta$ Cl-free+Iso) was used as the diagnostic parameter. The reference range was determined from measurements in cohorts of healthy controls $(n=84)$, obligate heterozygotes $(n=48)$ and patients with established CF ( $n=112) ; \Delta$ Cl-free+Iso was interpreted as normal $(<-12 \mathrm{mV})$, intermediate $(-12$ to $-7.7 \mathrm{mV})$, and abnormal $(>-7.7 \mathrm{mV}$ ) (figure 1).

\section{CFTR genotyping}

Extensive CFTR genotyping was performed in all subjects by multiplexed heteroduplex analysis followed by sequencing. ${ }^{15}$ Large deletions were detected using established conditions. ${ }^{16}{ }^{17}$

The 23 CFTR mutations recommended by the ACMG, were used as the initial screening test for both algorithms. For the American algorithm, the second step of CFTR

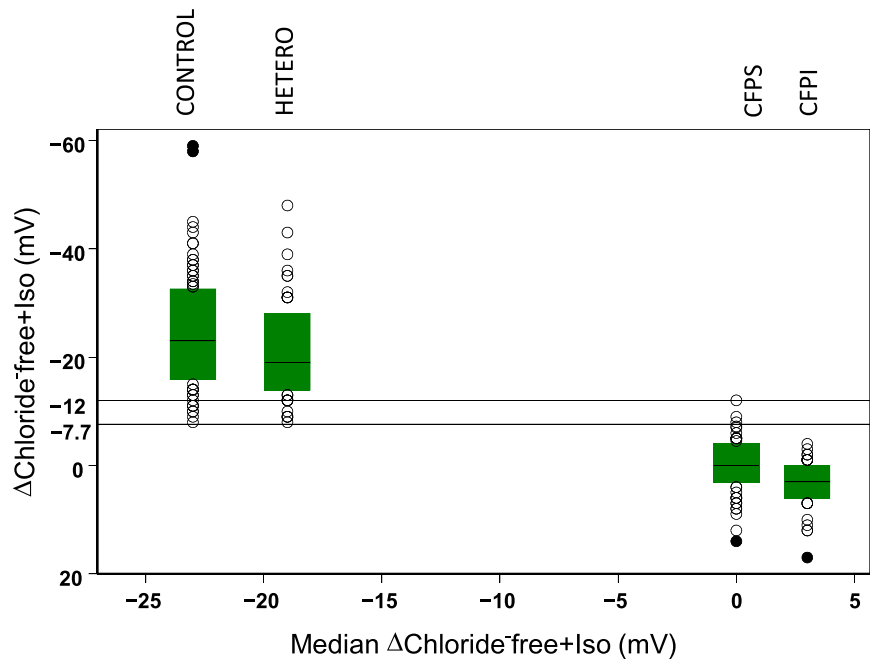

Figure 1 The reference range for the nasal potential difference (NPD) parameter of change $(\Delta)$ in cystic fibrosis transmembrane conductance regulator (CFTR)-mediated chloride diffusion following chloride-free and isoproterenol perfusion ( $\Delta$ Chloride-free+lso) was derived based on measurements in cohorts of healthy controls (CONTROL; $n=84$ ), obligate heterozygotes (HETERO; $n=48$ ) and patients with established CF with pancreatic sufficiency (CFPS; $n=64$ ) and insufficiency (CFPI; $n=43$ ). The range and relationship of each group with one another are demonstrated by arranging box plots for each group along the $x$ axis according to the value of the median for $\Delta$ Chloride-free+lso. Each box plot represents values within the 25th-75th percentiles (IOR). Values outside the 25th and 75th percentiles are represented by circles. Outliers, values that are more than 1.5 IQR above and below the 75th and 25th percentiles, respectively, are represented by solid circles. Horizontal dashed lines depict the medians of each group. Based on the range of overlap between CF and non-CF (CONTROL and HETERO) individuals, the reference range was as follows: normal $<-12 \mathrm{mV}$, borderline -12 to $-7.7 \mathrm{mV}$ and abnormal $>-7.7 \mathrm{mV}$. 
mutations included a conservative list of additional mutations which fulfilled the requirements of two consensus criteria as CF-causing mutations (figure 2, footnote). ${ }^{7}{ }^{18}$ For the
European diagnostic process, interpretation of results from extensive genotyping was based on the number of mutations identified.
Figure 2 Summary of the diagnostic algorithms for the American (A) and European guidelines (B). The diagnostic outcomes among the 208 subjects are also shown. The 23 mutations listed by the American College of Medical Genetics for population screening, was used as the initial screening panel for both diagnostic guidelines. ${ }^{9}$ The interpretation of extensive cystic fibrosis transmembrane conductance regulator (CFTR) mutation analyses differs between the American and European algorithms. For the American algorithm, the second step of CFTR mutations included additional mutations which fulfilled the requirements of two consensus criteria as CF-causing mutations. ${ }^{7}{ }^{18}$ For the European diagnostic process, interpretation of results from extensive genotyping was based on the number of mutations identified. CF-causing mutations on extensive mutation analysis were defined based on two consensus reports on interpretation of CFTR mutations. ${ }^{78}$ On this basis, CF-causing mutations include G85E, R117H(5T), R334W, R347P, A455E, I507del, F508del, G542X, G551D, R553X, R560T, $\mathrm{W} 1282 \mathrm{X}, \mathrm{N} 1303 \mathrm{~K}, 621+1 \mathrm{G}>\mathrm{T}, 711+1 \mathrm{G}>\mathrm{T}, 1717-$ $1 \mathrm{G}>\mathrm{A}, 2789+5 \mathrm{G}>\mathrm{A}, 3120+1 \mathrm{G}>\mathrm{A}, 3659$ delC, 3849 $+10 \mathrm{~kb} C>T, 2622+1 \mathrm{G}>\mathrm{A}, 2194 \mathrm{inA}, \mathrm{R} 1066 \mathrm{C}, 2752-$ $2 A>G, S 489 X, 1525-1 G>A, M 1101 K, 875+1 G>C$, 3272-26A $>$ G, I1234V, 758delC, E831X, R75X, S1251N, W679X, 2789+2 2789+3insA, -741T $>$ G, 1394delC, 4016insT, S549R (A>C), L218X, CFTRdele 4-10, R764X. NPD, nasal potential difference; Sw $\mathrm{Cl}^{-}$, sweat choride concentration.
A

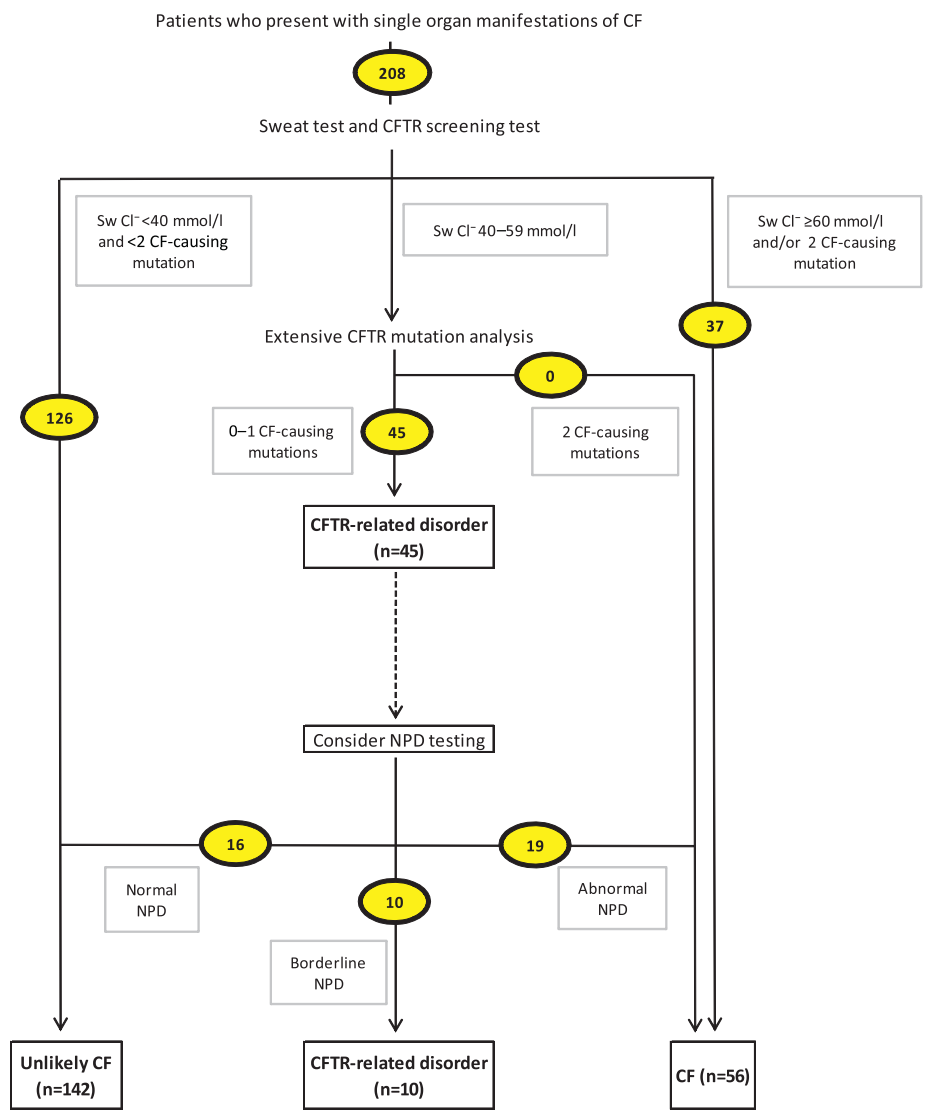

B Patients who present with single organ manifestations of $\mathrm{CF}$

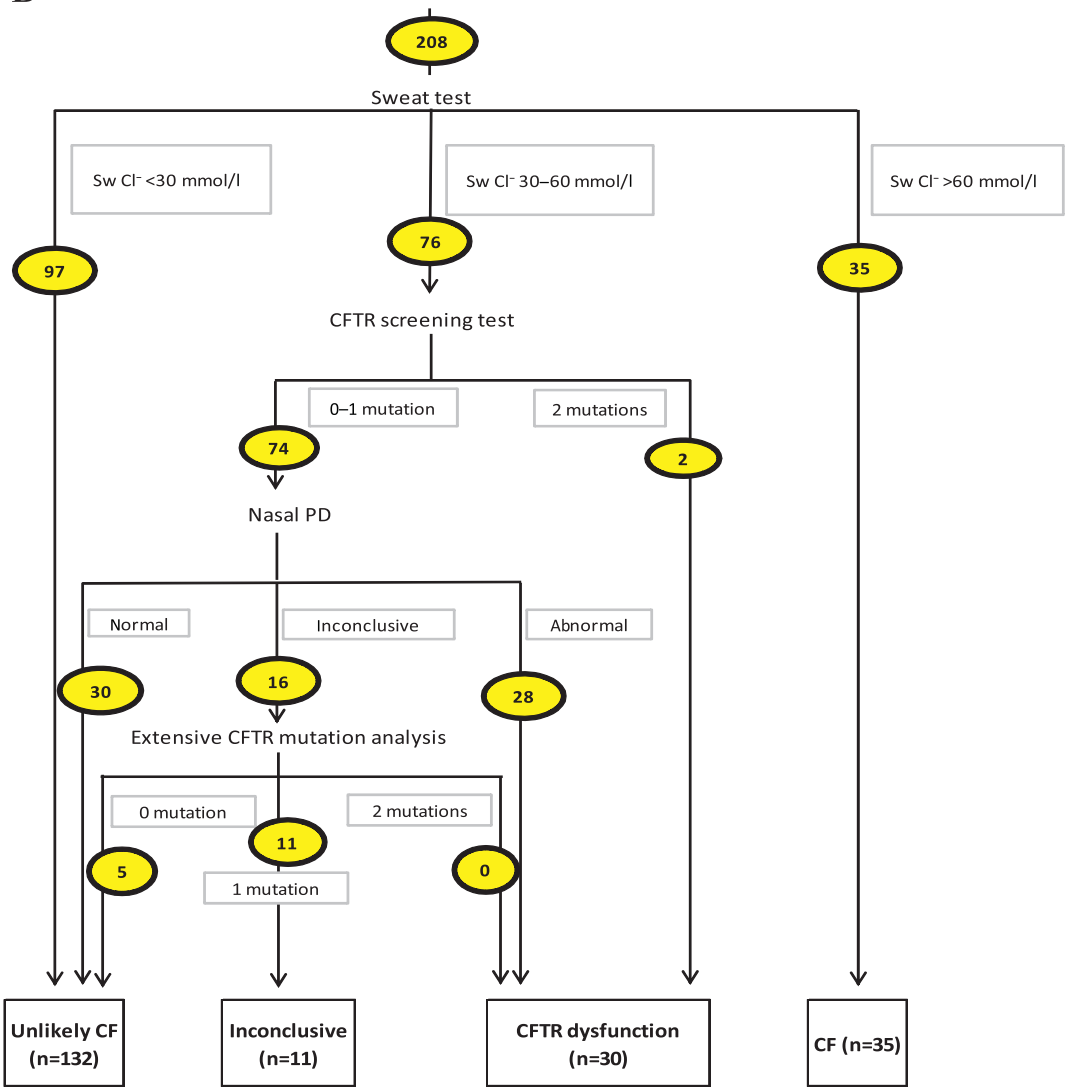




\section{Statistical analysis}

The American and European diagnostic processes were independently applied to all subjects. The European algorithm with sweat testing was used as the initial assessment (figure 2). Concordance between the two diagnostic algorithms was examined using observed agreement and $\kappa$ statistics. For the American algorithm, concordance was measured with and without NPD testing. The following assumptions were made regarding the differing terminologies used by American and European reports, respectively: 'CF' $=$ 'classic CF', 'CFTRrelated' $=$ 'CFTR dysfunction' and 'unlikely CF' $=$ 'unlikely CF'. Patients with an 'inconclusive' outcome in the European algorithm were excluded from concordance analyses. Observed agreement was calculated as the number of patients with the same diagnosis divided by the total number of patients. $\kappa$ values can range from -1 (complete disagreement) to 1 (perfect agreement), and interpreted by the degree of agreement: $\kappa<0.20$ is poor, $\kappa=0.21-0.40$ is fair, $\kappa=0.41-0.60$ is moderate, $\kappa=0.61-0.80$ is good and $\kappa=0.81-1.00$ is excellent. ${ }^{19}$

\section{RESULTS}

\section{Study population}

We prospectively recruited 208 subjects, consisting of $72(34.6 \%)$ RESP, 44 (21.2\%) PANC and 92 (44.2\%) AZOOSP. The mean age (SD; range) at the time of evaluation of RESP, PANC and AZOOSP subjects was 38.5 (15.9; 9.9-66.7), 24.3 (13.2; $7.9-59.9)$ and $34.8(5.3 ; 25.4-56.6)$ years, respectively. Fifty-one (70.8\%) RESP and 26 (59.1\%) PANC subjects were women. The number and type of identified mutations is summarised in table 1 and in the supplemental material.

\section{Diagnostic outcomes}

According to the American recommendations, 37 (17.8\%), 45 $(21.6 \%)$ and $126(60.6 \%)$ subjects could be diagnosed as having 'CF', 'CFTR-related disorder' and 'unlikely CF', respectively (table 2 and figure 2). Of note, second tier extensive genotyping for those with borderline sweat tests failed to confirm a ' $\mathrm{CF}$ ' diagnosis in any additional subjects. When NPD testing was incorporated into the American algorithm, 35 out of 45 patients with an initial outcome of 'CFTR-related disorder' were reclassified as either 'unlikely CF' $(n=16)$ or 'CF' ( $n=19)$. Among the 35 patients, there were 6, 2 and 27 RESP, PANC and AZOOSP subjects, respectively. Hence, when NPD was included, 56 $(26.9 \%), 10(4.8 \%)$ and $142(68.3 \%)$ subjects could be diagnosed as having 'CF', 'CFTR-related disorder' and 'unlikely CF', respectively.

Using the European algorithm, 35 (16.8\%), 30 (14.4\%), 11 $(5.3 \%)$ and $132(63.5 \%)$ patients were diagnosed as having 'classic CF', 'CFTR dysfunction', 'inconclusive' and 'unlikely CF', respectively (table 2 and figure 2). All 35 patients with

Table 1 Breakdown of number of CFTR mutations in all subjects and according to presenting phenotype

\begin{tabular}{lllll}
\hline & \multicolumn{2}{l}{ CFTR mutations } & \multirow{2}{*}{$\begin{array}{l}\text { Two CF-causing } \\
\text { mutations }\end{array}$} \\
\cline { 2 - 4 } & $\mathbf{0}$ & $\mathbf{1}$ & $\mathbf{2}$ & $10(4.8 \%)$ \\
All subjects $(\mathrm{n}=208)$ & $73(35.1 \%)$ & $44(21.2 \%)$ & $91(43.7 \%)$ & 10 \\
RESP $(\mathrm{n}=72)$ & $39(54.2 \%)$ & $17(23.6 \%)$ & $16(22.2 \%)$ & $4(5.6 \%)$ \\
PANC $(\mathrm{n}=44)$ & $22(50 \%)$ & $10(22.7 \%)$ & $12(27.3 \%)$ & $2(4.5 \%)$ \\
AZOOSP $(\mathrm{n}=92)$ & $12(13 \%)$ & $17(18.5 \%)$ & $63(68.5 \%$ & $4(4.3 \%)$ \\
\hline
\end{tabular}

AZOOSP, obstructive azoospermia; CFTR, cystic fibrosis transmembrane conductance regulator; PANC, idiopathic recurrent acute or chronic pancreatitis; RESP, idiopathic sinopulmonary disease.
Table 2 Diagnostic outcomes for all subjects classified by presenting phenotype according to the American and European consensus recommendations

\begin{tabular}{lcccc}
\hline & $\begin{array}{c}\text { All subjects } \\
(\mathbf{n = 2 0 8})\end{array}$ & $\begin{array}{l}\text { RESP } \\
(\mathbf{n = 7 2 )}\end{array}$ & $\begin{array}{l}\text { PANC } \\
(\mathbf{n}=\mathbf{4 4 )}\end{array}$ & $\begin{array}{l}\text { AZO0SP } \\
(\mathbf{n}=\mathbf{9 2})\end{array}$ \\
\hline American recommendations & & & & \\
Unlikely CF & $126(60.6 \%)$ & $50(69.5 \%)$ & $37(84.1 \%)$ & $39(42.4 \%)$ \\
CFTR-related disorder & $45(21.6 \%)$ & $8(11.1 \%)$ & $3(6.8 \%)$ & $34(37.0 \%)$ \\
CF & $37(17.8 \%)$ & $14(19.4 \%)$ & $4(9.1 \%)$ & $19(20.6 \%)$ \\
American recommendations with adjunctive NPD testing & & \\
Unlikely CF & $142(68.3 \%)$ & $54(75.0 \%)$ & $38(86.4 \%)$ & $50(54.3 \%)$ \\
CFTR-related disorder & $10(4.8 \%)$ & $2(2.8 \%)$ & $1(2.3 \%)$ & $7(7.6 \%)$ \\
CF & $56(26.9 \%)$ & $16(22.2 \%)$ & $5(11.5 \%)$ & $35(38.0 \%)$ \\
European recommendations & & & & \\
Unlikely CF & $132(63.5 \%)$ & $54(75.0 \%)$ & $35(79.6 \%)$ & $43(46.7 \%)$ \\
Inconclusive & $11(5.3 \%)$ & $1(1.4 \%)$ & $1(2.3 \%)$ & $9(9.8 \%)$ \\
CFTR dysfunction & $30(14.4 \%)$ & $3(4.2 \%)$ & $6(13.6 \%)$ & $21(22.8 \%)$ \\
Classic CF & $35(16.8 \%)$ & $14(19.4 \%)$ & $2(4.5 \%)$ & $19(20.7 \%)$ \\
\hline
\end{tabular}

AZOOSP, obstructive azoospermia; CF, cystic fibrosis; CFTR, cystic fibrosis transmembrane conductance regulator; NPD, nasal potential difference; PANC, idiopathic recurrent acute or chronic pancreatitis; RESP, idiopathic sinopulmonary disease.

'classic CF' would have been diagnosed as having 'CF' using American recommendations. Two subjects who were not diagnosed as having 'classic CF' by the European recommendations but considered to have 'CF' based on the American recommendation had PANC phenotypes, borderline sweat tests, and two CF-causing mutations (F508del/3849+10 kb C>T and sweat chloride $31 \mathrm{mmol} /$ litre; F508del/3659delC and sweat chloride $55 \mathrm{mmol} /$ litre).

Three (1.4\%) patients with pancreatic insufficiency (all RESP) were diagnosed as having 'CF' and 'classic CF' according to the American and European diagnostic algorithms, respectively.

\section{Concordance between American and European guidelines}

Concordance analysis between the American algorithm without NPD testing and the European algorithm with NPD testing was performed after excluding patients whose condition was labelled as 'inconclusive' by the European report. Observed agreement was $84.8 \%(167 / 197)$; that is $15.2 \% \quad(\mathrm{n}=30)$ patients had discrepant diagnoses. The corresponding $\kappa$ was 0.87 (95\% CI 0.82 to 0.92 ), suggesting an 'excellent' level of agreement (table 3). Discrepancies in 10 of 30 (33.3\%) subjects were due to differences in the lower limit of the borderline sweat chloride concentration (30 vs $40 \mathrm{mmol} / \mathrm{litre}$ ) (table 4). Extensive genotyping demonstrated that two and eight subjects carried one and two CFTR mutations, respectively. Nine of 10 patients were

Table 3 Concordance between the American and European consensus recommendations

\begin{tabular}{lcl}
\hline & Observed agreement, \% & $\kappa$ (95\% Cl) \\
\hline American (without NPD testing) versus European recommendations \\
All subjects & 84.8 & 0.87 (0.82 to 0.93$)$ \\
RESP & 90.2 & $0.92(0.87$ to 0.98$)$ \\
PANC & 86.1 & $0.65(0.36$ to 0.94$)$ \\
AZOOSP & 79.5 & $0.87(0.80$ to 0.94$)$ \\
American (with NPD testing) versus European recommendations \\
All subjects & 83.2 & $0.75(0.67$ to 0.83$)$ \\
RESP & 93.0 & $0.91(0.82$ to 0.99$)$ \\
PANC & 86.1 & $0.61(0.33$ to 0.89$)$ \\
AZOOSP & 73.5 & $0.65(0.53$ to 0.78$)$
\end{tabular}

AZOOSP, obstructive azoospermia; NPD, nasal potential difference; PANC, idiopathic recurrent acute or chronic pancreatitis; RESP, idiopathic sinopulmonary disease. 
Table 4 Summary of subjects with discrepant diagnostic outcomes due to sweat chloride concentrations of 30-39 mmol/litre

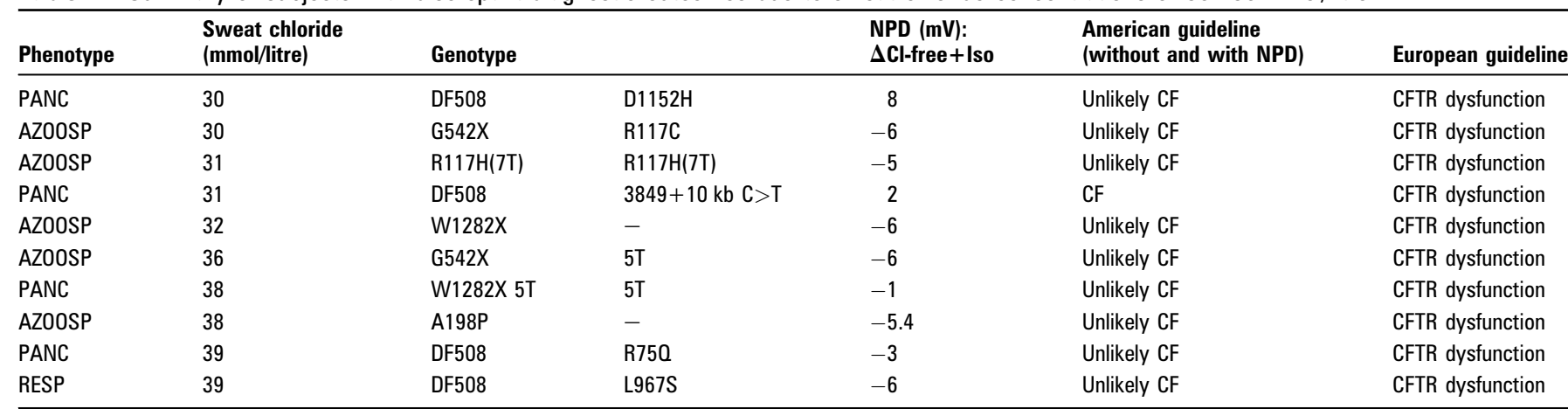

Change in CFTR-mediated chloride diffusion following perfusion with a chloride-free solution and isoproterenol $(\Delta \mathrm{Cl}$-free + Iso): normal $(<-12 \mathrm{mV})$, intermediate $(-12 \mathrm{to}-7.7 \mathrm{mV})$ and abnormal $(>-7.7 \mathrm{mV})$.

AZOOSP, obstructive azoospermia; CF, cystic fibrosis; CFTR, cystic fibrosis transmembrane conductance regulator; NPD, nasal potential difference; PANC, idiopathic recurrent acute or chronic pancreatitis; RESP, idiopathic sinopulmonary disease.

considered to have 'unlikely CF' according to the American criteria, whereas the European recommendations yielded a diagnosis of 'CFTR dysfunction'. According to the American criteria, the remaining subject (PANC) was diagnosed as having 'CF' due to the identification of two CF-causing mutations (F508del and $3849+10 \mathrm{~kb} \mathrm{C}>\mathrm{T})$. In contrast, this subject was considered to have 'CFTR dysfunction' by the European criteria due to borderline sweat chloride of $31 \mathrm{mmol} /$ litre and identification of two mutations following initial mutation screening. Discrepant diagnoses in the remaining 20 subjects were due to differences in interpreting genotypes and recommendations that led to NPD testing.

When NPD testing was incorporated into the American algorithm more subjects (33/197, 16.8\%) had discordant diagnoses than with sweat testing and genotyping alone. The observed agreement was 83.2\% (164/197) and can be interpreted as a 'good' level of agreement ( $\kappa=0.75$; $95 \%$ CI 0.67 to 0.83 ).

The degree of concordance varied according to phenotype (table 3), with the RESP phenotype demonstrating the greatest degree of concordance (>90\% observed agreement; 'excellent' agreement). The degree of concordance between the American and European guidelines did not change among RESP and PANC subjects when NPD testing was included in the American algorithm. Conversely, the level of agreement reduced from excellent to good for AZOOSP subjects when NPD testing was added to the American algorithm. Eleven patients whose condition was labelled as 'inconclusive' by the European criteria were categorised as having a 'CFTR-related disorder' $(n=7)$ and 'unlikely CF' $(n=4)$ by the American criteria (see online supplemental material). No subject was diagnosed as having 'CF' by the American criteria and conversely classified as 'unlikely CF' by the European guidelines, and vice versa.

\section{DISCUSSION}

There was 'good to excellent' concordance between the American and European recommendations. This is reassuring because the patient population we evaluated represents those that are most likely to be associated with diagnostic challenges. The greatest concordance was observed in subjects with the RESP phenotype, which is also reassuring since this phenotype is associated with the highest morbidity and mortality. In addition, all patients diagnosed as having 'classic CF' using European recommendations were classified as having ' $\mathrm{CF}$ ' by the American guidelines. No subject was diagnosed as having 'CF' by the American guideline but concurrently classified as 'unlikely CF' by the European recommendations, and vice versa. This outcome was not surprising for the following reasons: both guidelines universally accepted that sweat chloride concentrations $>60 \mathrm{mmol} /$ litre and $<30 \mathrm{mmol} /$ litre were associated with 'CF' and 'unlikely CF', respectively; we applied the same 23 mutations recommended by the ACMG to both algorithms; and extensive genotyping failed to confirm the diagnosis of $\mathrm{CF}$ in any patients with a normal or borderline sweat chloride concentration (American guidelines) despite expansion of the list of CF-causing mutations.

Nevertheless, there was a notable 'real-life' problem of discrepant diagnoses among $15 \%$ of subjects. One-third of the discrepant diagnoses were due to differences in the lower cutoff for sweat chloride concentration (30 vs $40 \mathrm{mmol} /$ litre) after 6 months of age. Mishra et $a l^{20}$ determined that the upper limit of the sweat chloride concentration in healthy 5-9 year olds was $39.5 \mathrm{mmol} /$ litre, which is consistent with the American cutoff of $40 \mathrm{mmol} /$ litre. In healthy subjects $\geq 10$ years old, the upper limit of sweat chloride concentration overlapped into the intermediate range. The lower cutoff $(30 \mathrm{mmol} /$ litre) recommended in the European guidelines was based on case observations that sweat chloride values $<40 \mathrm{mmol} /$ litre can occur in a small subgroup of individuals with $\mathrm{CF}^{36-821}$ The lower cutoff will subject a larger number of individuals without CF to diagnostic testing. In this study, there were nine subjects who would be discharged from the American algorithm as 'unlikely CF' due to sweat chloride $<40 \mathrm{mmol} /$ litre and the absence of two CFcausing mutations, but considered to have 'CFTR dysfunction' in the European algorithm. These nine individuals would possibly have a different clinical outcome and follow-up depending on which diagnostic guideline was applied. Since all nine subjects had abnormal NPD measurements in the CF range (table 4), the use of the higher intermediate sweat chloride cutoff of $40 \mathrm{mmol} /$ litre (in conjunction with mutation screening) may miss individuals who have CFTR-related disorders or CF. Nonetheless, it could be argued that the choice of lower cutoff merely represents an entry point into the diagnostic algorithm for patients who may otherwise be missed by sweat testing and mutation screening.

Discrepancies in the remaining subjects arose from differences in the recommended sequence and/or interpretation of genotyping and NPD testing. While the American guidelines recommend sweat chloride testing and screening as the most common CF-causing mutations at the first stage of testing, the European guidelines recommend sweat chloride testing (or genotyping) alone. It is well recognised that some confirmed CFcausing mutations can be associated with a normal or borderline 
sweat test (eg, $3849+10 \mathrm{~kb} \mathrm{C}>\mathrm{T}$ ). Thus, there is also the risk of a false-negative diagnostic outcome should sweat testing be performed in isolation. Extensive genotyping in both diagnostic algorithms did not significantly aid in the diagnosis of CF, consistent with findings from previous studies. ${ }^{36}$ Hopefully, the clinical utility of extensive genotyping will improve as more information (correlating specific mutations with functional and/or clinical data) emerges. ${ }^{22}$

Interestingly, the concordance between the American and European recommendations did not improve when NPD testing was included into the American algorithm, thus raising questions regarding the role of NPD. However, when NPD testing was incorporated into the American algorithm, 35 patients with an initial diagnosis of 'CFTR-related disorder' were reclassified as having 'CF' or 'unlikely CF'. Therefore, it is arguable that NPD testing played a role in clarifying the diagnosis of CF in these 35 patients, especially in patients with normal NPD results which makes the diagnosis of CF very unlikely. Falsepositive results can occur for reasons not intrinsic to the NPD test, including technically incorrect catheter location and perturbations of the nasal epithelium from allergies, infections and smoking. 3672324 However, the clinical utility of NPD (and alternative ex vivo intestinal current measurements ${ }^{25}$ ) remains limited by the lack of standardisation, validated reference values and a clear cutoff point for differentiating between individuals with CF and those with CFTR-related disorders. 72326

The vast majority of individuals in whom NPD testing suggested a diagnosis of CF were men with obstructive azoospermia (16 out of 19). Hence, clinical consideration is necessary concerning the diagnosis of CF in 'healthy' men with obstructive azoospermia. Follow-up should be offered to these patients because the presence of CFTR dysfunction may indicate the presence of subclinical disease and/or risk for future disease development in other affected organs (eg, pulmonary disease). Subclinical pulmonary disease has been reported in men with obstructive azoospermia associated with intermediate or abnormal sweat tests, ${ }^{27}$ but long-term pulmonary outcomes in this cohort are unknown.

Despite the 'good to excellent' concordance between the two guidelines, the use of different terminologies and definitions of disease is confusing for clinicians and patients, and may influence research study design and outcomes. When terminologies such as 'mild/non-classic/atypical' are used, there are risks of misinterpretation by both clinicians and patients as to whether an individual has CF disease or not, and false reassurance on the potential impact of disease since older patients presenting with broncho-pulmonary disease may progress to pulmonary failure and premature death or a life-saving lung transplant. ${ }^{72}$ Thus, there are benefits in having a unified guideline. To this end, a recent consensus document by European and North American experts recommended the term 'CFTR-related disorders' to describe subjects with CF-like manifestations in one or more organ, with evidence of CFTR dysfunction/mutation(s) that is insufficient to fulfil the current diagnostic criteria for $\mathrm{CF}^{26}$

The limitations of this study include lack of follow-up clinical outcomes and repeat diagnostic test results, and limiting enrolment to older children and adults. Borderline sweat test results are known to occur in asymptomatic newborn screen-positive infants giving rise to considerable diagnostic uncertainty. ${ }^{29}$ Another potential limitation was the use of two different sweat test techniques, which occurred due to a change in technique by our laboratory. We demonstrated that sweat chloride concentrations measured by Macroduct highly correlated with the Gibson and Cooke method in all ranges, including values in the intermediate range $(\mathrm{r}=0.93, \mathrm{p} \leq 0.0001) .{ }^{30}$ Hammond et al made similar conclusions in a large number of healthy controls and CF subjects. ${ }^{31}$ Furthermore, in a subanalysis of individuals with sweat chloride concentrations $<60 \mathrm{mmol} /$ litre, the $95 \% \mathrm{CI}$ reduced to $\pm 13 \mathrm{mmol} /$ litre, well within the range recommended by the Clinical and Laboratory Standards Institute. ${ }^{32}$

To conclude, despite differences between the American and European diagnostic recommendations, statistical analyses demonstrated 'good to excellent' concordance among subjects who present with single organ manifestations of CF. Concordance was excellent among subjects with chronic sinopulmonary disease. Approximately $15 \%$ of patients had discordant diagnoses. While, diagnostic algorithms support a rigorous approach to the evaluation of complex diseases such as CF, they should be regarded as general guidelines rather than dogma. We encourage efforts towards developing unified consensus diagnostic criteria for patients with $\mathrm{CF}$.

Acknowledgements The authors thank the many research subjects who gave up their time to participate in the project. The authors acknowledge the support and assistance of Louise Taylor, Susan Carpenter, Thora St Cyr, Rachel Paul, Debbie Ryan, Lenny Chong, Leia Spencer, Xiao-Wei Yuan, Qiuju Huang, Satti Beharry and Wan Ip.

Contributors No undisclosed writing assistance was sought. CYO: study design, data acquisition, data analysis and interpretation, statistical analysis, and drafting of manuscript. $\mathrm{AD}$ : data analysis and interpretation, statistical analysis. LE: subject recruitment, data acquisition. KJ: study conception and data acquisition. SM: subject recruitment, data acquisition. TG: study conception and critical revision of manuscript RD: technical and material support, data interpretation, and critical revision of manuscript. PK: study conception and data acquisition. MS: study conception and data acquisition. ET: study conception, data acquisition, and critical revision of manuscript. PRD: study conception and design, obtained funding, data analysis and interpretation, drafting of manuscript, study supervision.

Funding PD and ET were supported by research grants from the Canadian Cystic Fibrosis Foundation and Genome Canada through the Ontario Genomics Institute as per research agreement 2004-0Gl-3-05, the Ontario Research Foundation and from the Lloyd Carr-Harris Foundation. CYO and TG were funded by the Canadian Cystic Fibrosis Foundation Fellowship Awards and CYO received a Canadian Child Health Clinician Scientist Program Career Enhancement Award. RD was funded by a CIHR-Ontario Women's Health Council joint Fellowship.

\section{Competing interests None.}

Ethics approval Ethics approval was provided by the ethics boards of the Hospital for Sick Children, St Michael's Hospital and Mt Sinai Hospital, Toronto.

Provenance and peer review Not commissioned; externally peer reviewed.

\section{REFERENCES}

1. Sharer N, Schwarz M, Malone G, et al. Mutations of the cystic fibrosis gene in patients with chronic pancreatitis. N Engl J Med 1998;339:645-52.

2. Ooi CY, Dorfman R, Cipolli M, et al. Type of CFTR mutation determines risk of pancreatitis in patients with cystic fibrosis. Gastroenterology 2011;140:153-61.

3. Bishop MD, Freedman SD, Zielenski J, et al. The cystic fibrosis transmembrane conductance regulator gene and ion channel function in patients with idiopathic pancreatitis. Hum Genet 2005:118:372-81.

4. Girodon E, Cazeneuve C, Lebargy F, et al. CFTR gene mutations in adults with disseminated bronchiectasis. Eur J Hum Genet 1997;5:149-55.

5. Jarvi K, Zielenski J, Wilschanski $\mathrm{M}$, et al. Cystic fibrosis transmembrane regulato and obstructive azoospermia. Lancet 1995;345:1578.

6. Wilschanski M, Dupuis A, Ellis $L$, et al. Mutations in the cystic fibrosis transmembrane regulator gene and in vivo transepithelial potentials. Am J Resp Crit Care Med 2006;174:787-94.

7. Farrell PM, Rosenstein BJ, White TB, et al. Guidelines for diagnosis of cystic fibrosis in newborns through older adults: Cystic Fibrosis Foundation Consensus Report. J Pediatr 2008;153:S4-14.

8. De Boeck K, Wilschanski M, Castellani C, et al. Cystic fibrosis: terminology and diagnostic algorithms. Thorax 2006;61:627-35.

9. Watson MS, Cutting GR, Desnick RJ, et al. Cystic fibrosis population carrier screening: 2004 revision of American College of Medical Genetics mutation panel. Genet Med 2004;6:387-91.

10. Goubau C, Wilschanski M, Skalická V, et al. Phenotypic characterization of patients with intermediate sweat chloride values: towards validation of the European diagnostic algorithm for cystic fibrosis. Thorax 2009;64:683-91. 
11. Gonska T, Choi P, Stephenson A, et al. Role of cystic fibrosis transmembrane conductance regulator (CFTR) in patients with chronic sinopulmonary disease. Chest. Published Online First: 15 Mar 2012. doi:10.1378/chest.11-2543.

12. Gibson LE, Cooke RE. A test for concentration of electrolytes in sweat in cystic fibrosis of the pancreas utilizing pilocarpine by iontophoresis. Pediatrics 1959:23:545-9.

13. Hammond KB, Turcios NL, Gibson LE. Clinical evaluation of the Macroduct sweat collection system and conductivity analyzer in the diagnosis of cystic fibrosis. $J$ Pediatr 1994:124:255-60.

14. Knowles MR, Paradiso AM, Boucher RC. In vivo nasal potential difference: techniques and protocols for assessing efficacy of gene transfer in cystic fibrosis. Hum Gene Ther 1995:6:445-55.

15. Zielenski J, Aznarez I, Onay T, et al. CFTR mutation detection by multiplex heteroduplex (mHET) analysis on MDE gel. Methods Mol Med 2002;70:3-19.

16. Ferec C, Casals $\mathrm{T}$, Chuzhanova $\mathrm{N}$, et al. Gross genomic rearrangements involving Deletions in the CFTR gene: characterization of six new events from a large cohort of hitherto unidentified cystic fibrosis chromosomes and meta-analysis of the underlying mechanisms. Eur J Hum Genet 2006:14:567-76.

17. Audrezet MP, Chen JM, Raguenes 0 , et al. Genomic rearrangements in the CFTR gene: extensive allelic heterogeneity and diverse mutational mechanisms. Hum Mutat 2004:23:343-57.

18. Castellani C, Cuppens $\mathrm{H}$, Macek M Jr, et al. Consensus on the use and clinical interpretation of cystic fibrosis mutation analysis in clinical practice. J Cystic Fibrosis 2008;7:179-96

19. Landis JR, Koch GG. The measurement of observer agreement for categorical data. Biometrics 1977:33:159-74.

20. Mishra A, Greaves R, Smith K, et al. Diagnosis of cystic fibrosis by sweat testing age-specific reference intervals. J Pediatr 2008;153:758-63.

21. Bienvenu T, Sermet-Gaudelus I, Burgel PR, et al. Cystic fibrosis transmembrane conductance regulator channel dysfunction in non-cystic fibrosis bronchiectasis. $\mathrm{Am} \mathrm{J}$ Respir Crit Care Med 2010:181:1078-84.
22. Clinical and Functional Translation of CFTR. Baltimore: John Hopkins University. http://www.cftr2.com (accessed 5 Apr 2012).

23. Cantin AM, Hanrahan JW, Bilodeau, et al. Cystic fibrosis transmembrane conductance regulator function is suppressed in cigarette smokers. Am J Respir Crit Care Med 2006;173:1139-44.

24. Middleton PG, House HH. Measurement of airway ion transport assists the diagnosis of cystic fibrosis. Pediatr Pulmonol 2010:45:789-95.

25. Veeze HJ, Sinaasappel M, Bijman J, et al. Ion transport abnormalities in rectal suction biopsies from children with cystic fibrosis. Gastroenterology 1991:101:398-403.

26. Bombieri C, Claustres M, De Boeck K, et al. Recommendations for the classification of diseases as CFTR-related disorders. J Cyst Fibros 2011;10(Suppl 2):S86-102.

27. Gilliam M, Moltyaner Y, Downey GP, et al. Airway inflammation and infection in congenital bilateral absence of the vas deferens. Am J Respir Crit Care Med 2004:169:174-9.

28. Gilljam K, Ellis L, Corey $\mathrm{M}$, et al. Clinical manifestations of cystic fibrosis among patients diagnosed in adulthood. Chest 2004;126:1215-24.

29. Borowitz D, Parad R, Sharp JK, et al. Cystic Fibrosis Foundation practice guidelines for the management of infants with cystic fibrosis transmembrane conductance regulator-related metabolic syndrome during the first two years of life and beyond. J Pediatr 2009:155:S106-16.

30. Rose JB, Ellis J, John B, et al. Does the Macroduct ${ }^{\circledR}$ collection system reliably define sweat chloride concentration in subjects with intermediate results? Clin Biochem 2009:42:1260-4

31. Hammond KB, Nelson MS, Turcois L, et al. Clinical evaluation of the Macroduct sweat collection system and conductivity analyzer in the diagnosis of cystic fibrosis. J Pediatrics 1994:124:255-60.

32. Clinical Laboratory Standards Institute formerly National Committee for Clinical Laboratory Standards. Sweat Testing: Sample Collection and Quantitative Analysis: Approved Guideline: National Committee for Clinical Laboratory Standards Wayne, PA: Clinical Laboratory Standards Institute, 2000. NCCLS Document C34-A2

\section{Have confidence in your decision making.}
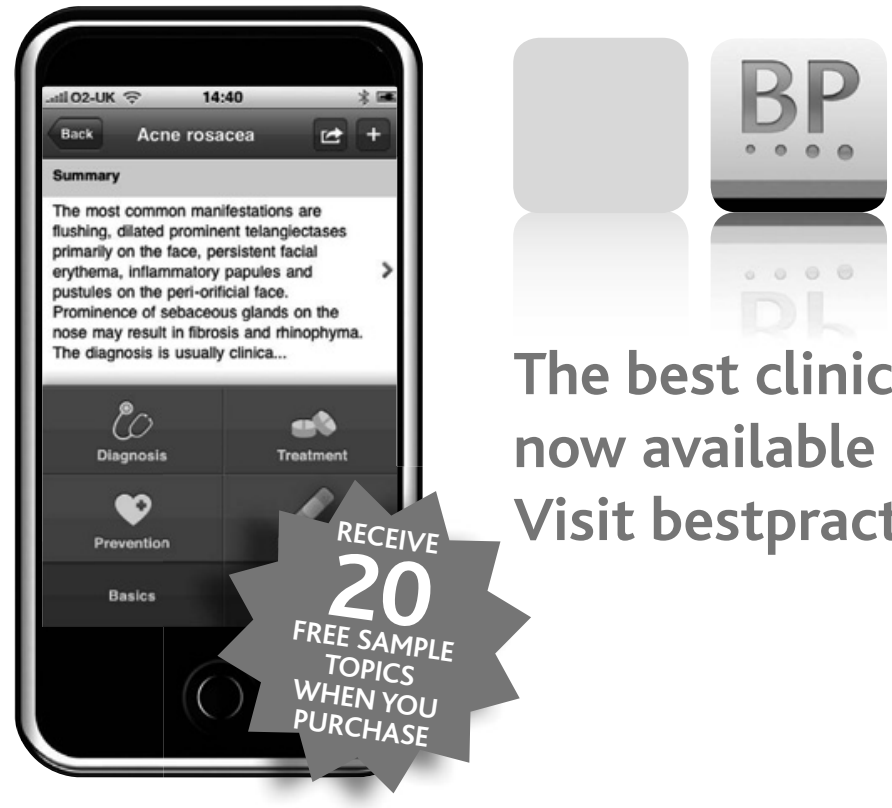

The best clinical decision support tool is now available as an app for your iPhone. Visit bestpractice.bmj.com/app

\section{clinicians $\bullet$ medical students $\bullet$ nurses $\bullet$ healthcare practitioners}

